\title{
SOLAR TRACKING SYSTEM
}

\author{
${ }^{1}$ Ankit Kumar Yadav, ${ }^{2}$ Naman Rakesh, ${ }^{3}$ Saksham, Yadav, ${ }^{4}$ Prof. R. S. Ambekar \\ Department of Electrical Engineering \\ Bharati Vidyapeeth (Deemed to be University) College Of Engineering, Pune
}

\section{ABSTRACT:}

People of today's world are more concerned about the environmental changes occurring due to pollution and depletion of natural occurring fossil fuels. The solar energy plays a major role in reducing the above stated concerns. The society seekers are educating themselves day by day. Due to the reason they are focusing on renewable energy resources. But the currentt solar photoelectric conversion efficiency is gradually low. The technology used in tracker can improve the rate of conversion of solar energy, so the dualaxial memory stored controller is shown in this research paper and detailed analysis of the same has been illustrated, solar tracking system based on the quadrant location phenomena is designed in order to enhance the efficiency. The above stated system is of high precision, simple structure, easy operation, etc. This paper also tries to increase power generation by placing the equipment such that, it gets maximum sunlight automatically throughout the day. This system tracks maximum intensity of light all the day along. When there is decrease in intensity of sunlight, this system automatically changes its position to get maximum intensity of light.

\section{KEYWORDS- Stepper Motor, LDR's, Solar Panel, Driver IC, Atmega-328, Sensors}

\section{I.INTRODUCTION}

A solar tracking system is a device onto which solar panels are placed, which tracks the rotation of the sun movement across the sky, and keeps note that the maximum light intensity should be incident on the panels all the day along. When the sun light starts falling, the tracking system will try to move through the path so the best amount of sunlight is incident on the panels. The tracking system is made such that it can solve the problems which are in static panels. It is completely automatic and moves the panel such that it should always be in front of sun until that is visible. The best feature of this system is that instead of taking the earth as its reference, it takes the sun as its source. Its sensors constantly track the sunlight and move the panels towards the area where the efficiency of sunlight is maximum and rotate the panel towards the direction where the intensity of sunlight is maximum.

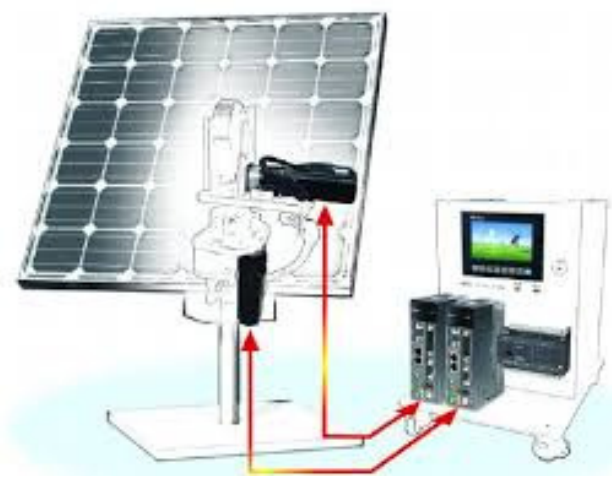

- Fig. 1: Solar tracking system

Position of the sun changes with season and also day time during the motion in sky. the solar tracking comprises of two types i.e., single and dual axis. There are two possible axis in the single axis solar tracker either horizontal axis or vertical. Generally, horizontal axis adopted in those areas where there is sun at top, day duration are very less. The vertical axis which is adopted in high altitudes where duration of day are high.

Dual axis tracking system have both horizontal axis as well as vertical axis and is able to point the location and it's motion anywhere in the 
sky. This is used in control system of astronomical telescope.

Sun tracking drives, is classified into three categories on the basis of type of drives, sensors, positioning system

- Passive trackers- use the sun radiation for gas heating which moves the tracking system throughout the sky.

- Active trackers- use the electrical and gear system to move the tracker.

- Open loop Tracker- In this no sensors are used but sun's position set by using pre recorded data for specific site.

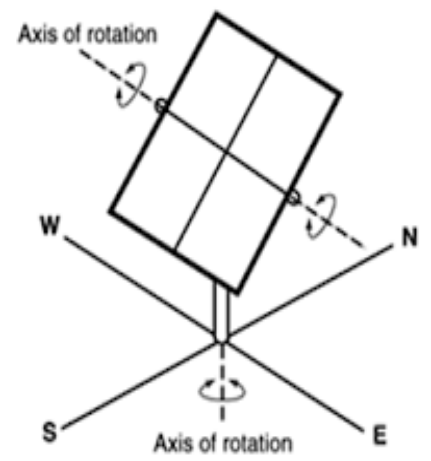

Two-axis tracking system

Dual Axis Solar Tracker

\section{DESIGN OF PROPOSED DUAL AXIS SOLAR TRACKING SYSTEM}

The proposed device should follow certain parameters and here are those parameters-

1. It should be reliable under harsh conditions like change in temperature, wind, dust, and humidity.

2. It should consume minimum electricity so that there can be optimum performance to cost ratio.

3. As solar panels are very costly, the tracker should give good results with small investment and should have high efficiency.

4. Taking into account all the parameters the proposed plan to drive the solar panel is based on below mentioned components.
5. A stepper motor, light dependent resistors, potentiometers for speed control and tolerance, ATmega328 microprocessor based arduino uno .

6. A system is provided for the measurement of intensity of solar light to the photovoltaic cells, representing the sensor which gives commands to the solar panel.

\section{KIT COMPONENT SPECIFICATIONS}

\section{a. Stepper Motor}

Holding Torque- $42 \mathrm{~N}-\mathrm{cm}$

Detent Torque- $1.5 \mathrm{~N}-\mathrm{cm}$

Rotor Interia- $0.057 \mathrm{~kg}-\mathrm{cm}^{\wedge} 2$

Weight-230 gm

Phase currentt- $1.5 \mathrm{~A}$

Phase Resis- $2.1 \Omega$

Phase Inductance- $5 \mathrm{mH}$

\section{b. Atmega 328}

Risc Architecture

4/8/16/32 Kb Flash Memory

256/512/1 Kb EEPROM

Operating Vtg-5 V

Temp. Range- -40

c. Driver IC

Power Supply Voltage- -0.3-47V

Storage Temperature Range- $-60-150^{\circ} \mathrm{C}$

\section{d. Solar Panel}

Nominal vtg-12V

No of cells- 36

Max power currentt- $0.58 \mathrm{~A}$

Open ckt vtg- $10.5 \mathrm{~V}$

Short ckt currentt-0.65A

\section{DESIGN \& SETUP}

LDRs are mounted on each corner of panels which works in pair for dual axis arrangement. These LDRs give signal to the ADC channels of atmega328.This ADC channels convert to digital signals and compares. The pair havinng less resis value will give higher currentt signal to the motor driver IC. Driver IC amplifies the incoming signals and fed to stepper motor( which rotates both horizontally and vertically as it is a dual axis tracker ). Stepper motor rotates in the direction of pair of LDRs 
havinng less resis value along with panel. Voltage and temperature sensors also fitted on solar panel and these values are displayed on LCCD. Whole setup work on $12 \mathrm{v}$ supply

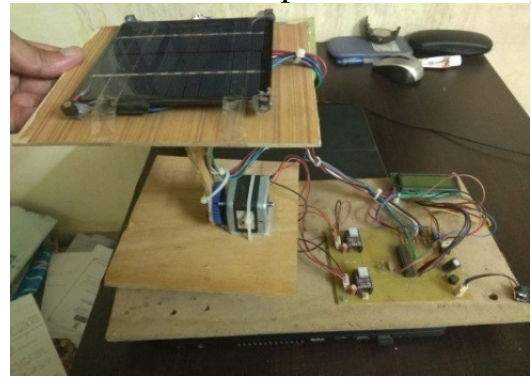

Actual Model of Project

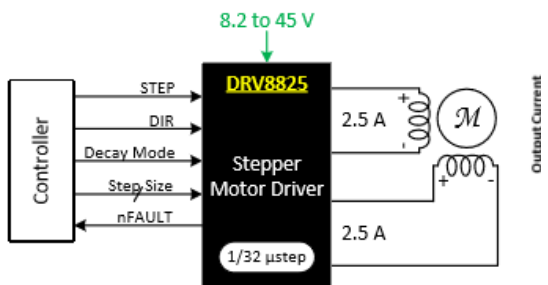

Driver IC

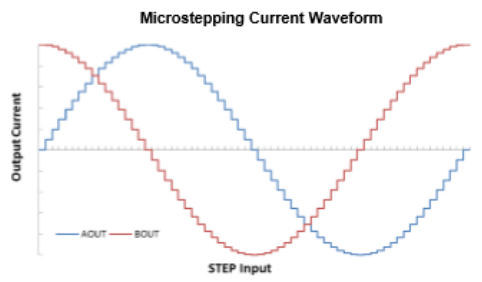

\section{v. SOURCE CODE \\ Waveform Graph}

\#include <LiquidCrystal.h>

\#define diR11 2

\#define steps1 3

\#define dir2 7

\#define steps2 8

int ltop $=\mathrm{A} 2$;

int rtop $=A 1$;

int 1 btm $=A 4$;

int rbtm $=\mathrm{A} 3$;

int $i, j$;

int speedd $=80$;

int sensorPin $=\mathrm{A} 5 ; / / \mathrm{sp}$

const int sensor=A0; / / temp

int analogInput $=5$;

float pv_r=23.40;//r

float currentt $=0.00$;

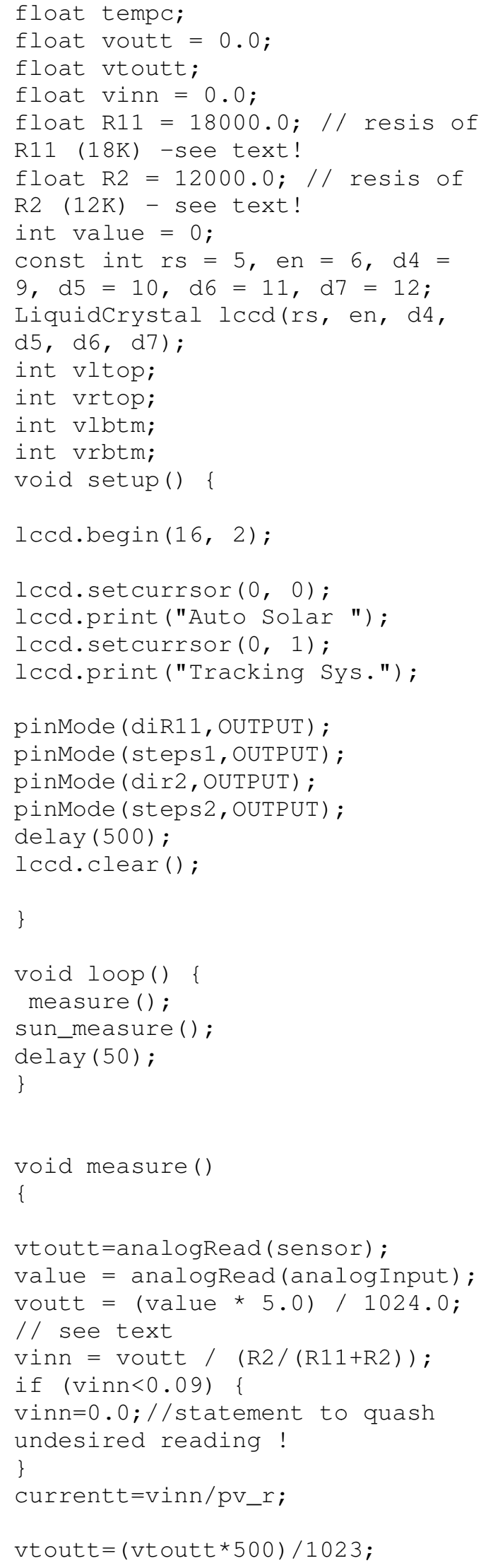




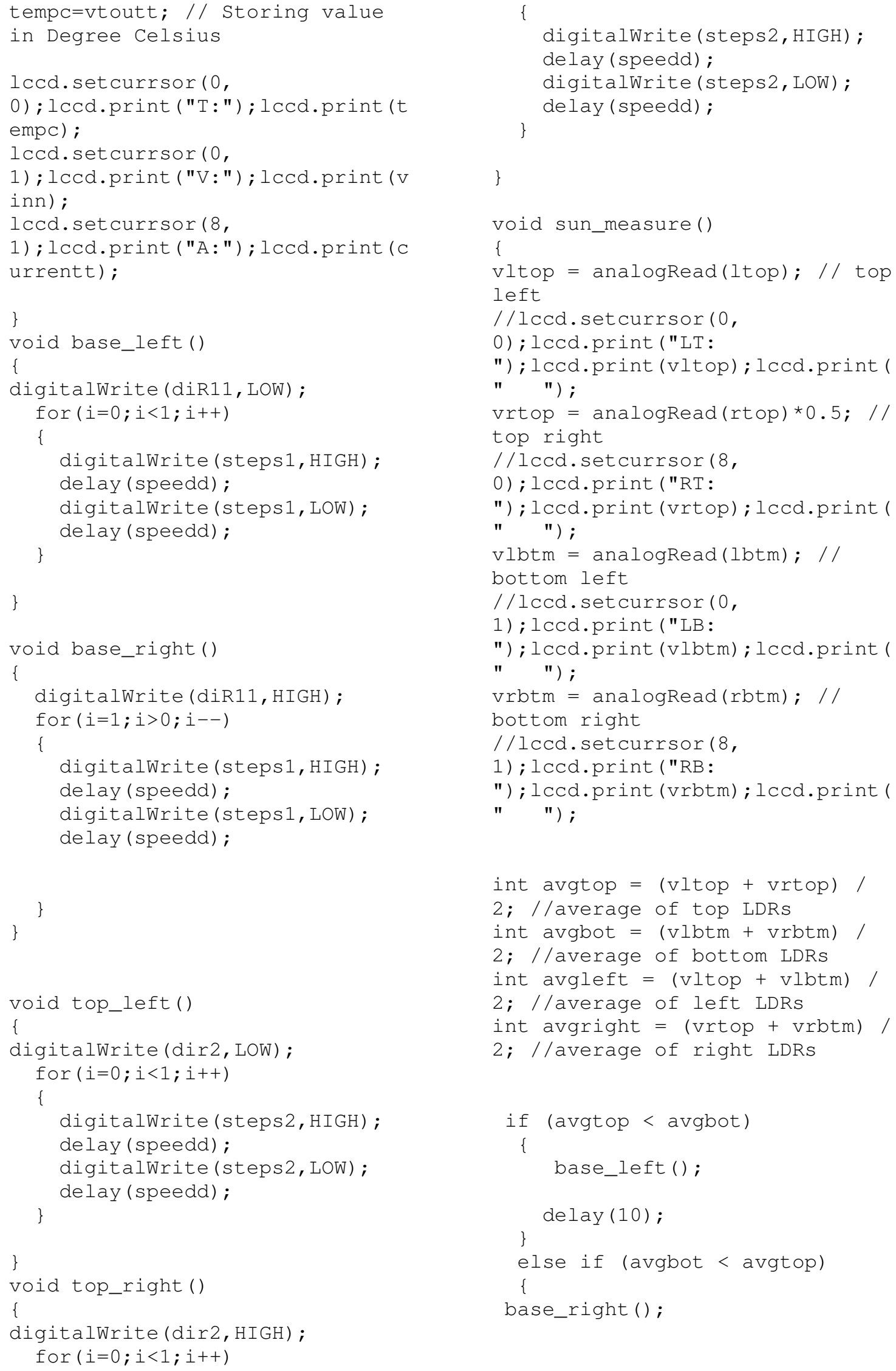




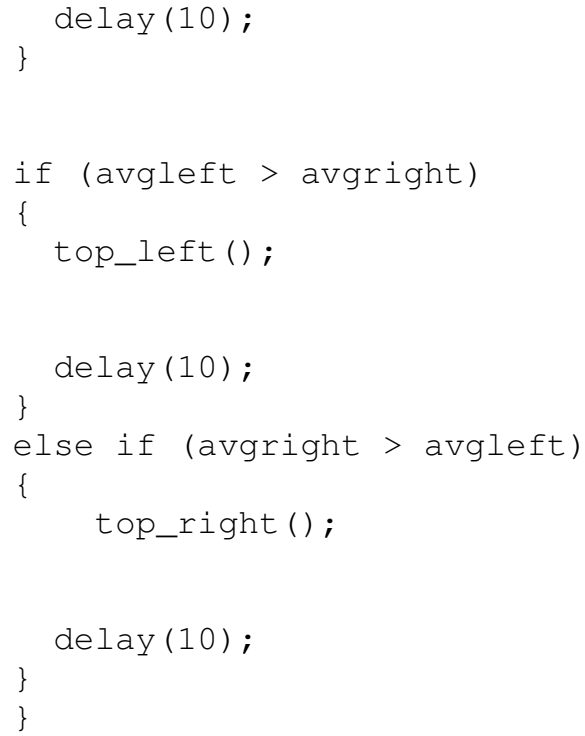

\section{CONCLUSION}

In this paper we presented and showed how we can increase the frequency of photovoltaic cells using solar tracking system over static solar panels. In this paper we showed that using automated solar tracker we can increase efficiency upto $30 \%-40 \%$. This is quite magnificient to achieve as non-renewable resources are depleting day by day and in future we need alternatives for them, so this is one of the most efficient method to cope up which such problems.

\section{REFERENCE}

[1] Energy and electrical Engineering and Management - Amlaan Chakraborty PHI.[2003]

[2] Energy: Management, generation, Supply and conservation - Dr. Clivi Biggs.[2006]

[3] Energy Conservation: Success and unsuccess - John Sawhil, Richard Cotton Brooklyn Institution Press.

[4] Handbook of Energy Conservation - H.M. Robert Downet Jr., J.M. Collins - Alken Publishing Unit.

[5] Electric Machines -Dr. D.P.Kothary, I.J. Nagraath - Tata Mc.Graw Hill [2014] 